\title{
The Influence of Patterned Nanofiber Meshes on Human Mesenchymal Stem Cell Osteogenesis
}

\author{
Albino Martins, ${ }^{*}$ Marta L. Alves da Silva, Susana Faria, \\ Alexandra P. Marques, Rui L. Reis, Nuno M. Neves
}

A specially designed electroconductive collector enables the electrospinning of P-NFM composed of areas of parallel/uniaxially aligned fibers and areas of random/orthogonal nanofiber distribution. The biological relevance of P-NFM is demonstrated using hBMSCs as an autologous cell source. The structures induce cell orientation along the uniaxially aligned fibers, mainly during earlier culturing periods under basal and osteogenic differentiation conditions. The microtopography of the P-NFM also controls the deposition of mineralized extracellular matrix along the pre-defined fiber direction. Genotypic characterization confirms the successful differentiation into the osteogenic lineage.

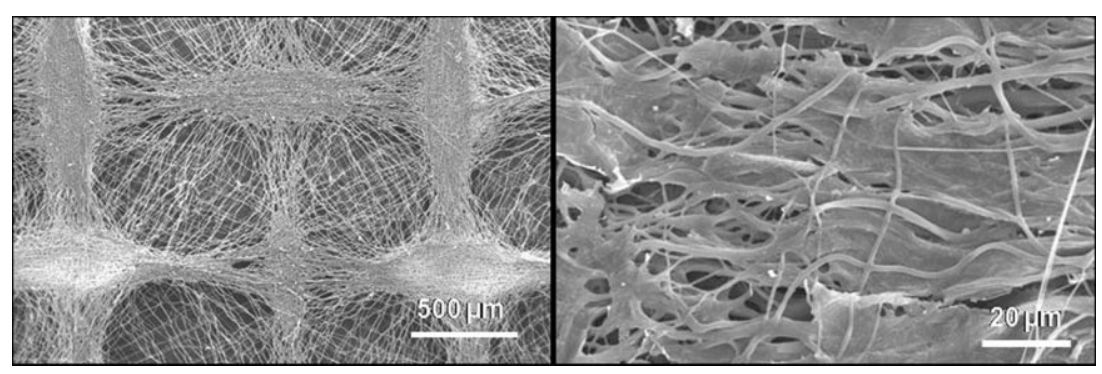

\section{Introduction}

In the tissue engineering field, electrospinning has received considerable interest and research effort as a

A. Martins, M. L. Alves da Silva, A. P. Marques, R. L. Reis, N. M. Neves

3B's Research Group, Biomaterials, Biodegradables and Biomimetics; Department of Polymer Engineering, University of Minho; Headquarters of the European Institute of Excellence on Tissue Engineering and Regenerative Medicine, AvePark, Zona Industrial da Gandra; S. Claudio do Barco; 4806-909 Caldas das Taipas, Guimarães; Portugal

E-mail: amartins@dep.uminho.pt

A. Martins, M. L. Alves da Silva, A. P. Marques, R. L. Reis, N. M. Neves

IBB - Institute for Biotechnology and Bioengineering, PT Associated Laboratory, Braga, Portugal

S. Faria

Research Center Officina Mathematica, Department of Mathematics for Science and Technology, University of Minho,

Campus de Azurém, 4800-058 Guimarães, Portugal polymeric nanofibrous scaffold production technique. ${ }^{[1-5]}$ The obtained mesh-like structures are typically characterized by a random distribution of fibers with diameters in the nanometer/sub-micrometer range and, thus, by a high specific surface area. The topography and surface morphology of this nanofibrous scaffold, resembling the fibrous structure of the natural extracellular matrix (ECM) of many living tissues, has been considered a major benefit of these structures. ${ }^{[4]}$ High microporosity and improved mechanical properties are also important properties of electrospun nanofiber meshes, allowing mass transport across the structure without compromising its structural integrity. In particular, these properties seem to be beneficial in the adhesion, viability, proliferation and maturation or differentiation of different cell types, namely keratinocytes and fibroblasts, ${ }^{[6]}$ smooth muscle and endothelial cells, ${ }^{[7]}$ chondrocytes, ${ }^{[8,9]}$ osteoblasts, ${ }^{[10,11]}$ cardiomyocytes, ${ }^{[12]}$ mesenchymal stem cells ${ }^{[13]}$ and neural stem cells. ${ }^{[14]}$ Indeed, such fiber meshes offer specific properties, as previously mentioned, for inducing tissue regeneration, once cells can bridge the 
scaffold pores and fibers, allowing faster and more homogeneous tissue growth.

Functional and structural engineering of musculoskeletal tissue may involve the use of appropriated cells cultured with specific growth factors in biomaterial scaffolds. ${ }^{[15-17]}$ While several tissues remain an important source of therapeutic relevant differentiated cells, stem cells have emerged as a strong alternative due to their expansion potential and the fact that they can be obtained from autologous sources. ${ }^{[18]}$ Additionally, advances in stem cell biology have shown that mesenchymal stem cells (MSCs) can differentiate into a variety of connective tissues, including bone, cartilage, fat, muscle and tendon, when cultured with appropriated supplemented culture media and specific environments. ${ }^{[19]}$ Moreover, the successful differentiation of MSCs along these distinct lineages on electrospun nanofibrous scaffolds has been also demonstrated. ${ }^{[13,20-22]}$

In the electrospinning technique, a high voltage potential is applied to a polymeric solution held in a syringe and coupled to a pump for flow control. A jet is driven from the needle in the direction of the grounded collector. The large diameter reduction from a millimeter fluid jet to a nanoscale solid fiber is due to an instability, where the jet is stretched by fast whipping and bending, in the way towards the collector, also involving solvent evaporation. ${ }^{[23]}$ Thus, the polymeric solution properties (i.e., concentration, viscosity, surface tension, conductivity and type of solvent), the processing parameters (i.e., electric field, needle tip-to-collector distance and polymeric flow rate) and the environmental conditions are all determinant factors for a successful electrospinning process. ${ }^{[24,25]}$ Among them, the type of collector may play an important role in the production of nanofiber meshes with different topographies. Typically, electrospun nanofibers are collected randomly in a mesh-like structure due to the bending instability associated with the electrified polymeric jets. The main topographical achievement reported in the literature is the production of parallel alignment of nanofibers in meshes. ${ }^{[24,26]}$ These meshes could be produced using rotating cylinder or plate collectors, conductive metallic strips with a geometrical distribution and sharp metallic pieces positioned oppositely. However, the production of electrospun nanofiber meshes with complex ordered topographies and patterns remains a challenge. ${ }^{[27]}$ Recently, our group and others described the possibility of producing electrospun nanofiber meshes comprising both types of topographies (random and parallel alignment of nanofibers in the same mesh), designated as patterning of polymer nanofiber meshes. ${ }^{[28-31]}$

We report herein the production of patterned nanofiber meshes (P-NFM) by electrospinning, using a specially designed electroconductive collector, obtaining a chesslike geometry. These nanofibrous structures are characterized by areas of random orthogonal alignment and areas of parallel uniaxial alignment of fibers in the same mesh. It is frequently mentioned in the literature that the typical random distribution of electrospun nanofibers in a meshlike structure resembles the topography of the native collagen ECM of various connective tissues. However, the complex ordered organization of the ECM is not usually replicated in this typical randomly aligned nanofibrous structure. Consequently, we aim to take a step forward in the nanofabrication of structures by mimicking more closely the structural complexity of the natural ECM. Although the processing parameters controlling the deposition and arrangement of fibers on the patterned architectures are well documented, ${ }^{[30]}$ the potential of patterned nanofiber meshes in the biomedical field has not yet been sufficiently explored. Therefore, this manuscript represents the first research study on the potential of P-NFM for sustaining the differentiation of relevant human stem cells for tissue engineering and regenerative medicine applications. To test the potential of these structures for bone tissue engineering, human bone marrow mesenchymal stem cells (hBMSCs) were seeded on and induced to differentiate towards the osteogenic lineage. This cellular performance (i.e., adhesion, morphology, viability, proliferation and differentiation) was assessed at different points in time to understand the influence of patterned nanofiber meshes, compared to the typical random nanofibrous structures produced by electrospinning.

\section{Experimental Section}

\section{Production of Patterned Nanofiber Meshes}

A polymeric solution of poly( $\varepsilon$-caprolactone) (PCL, TONE ${ }^{\mathrm{TM}}$, Union Carbide Chemicals and Plastics Division, New Jersey) at 17\% (w/v) was prepared using a mixture of chloroform and dimethylformamide (7:3), as described elsewhere. ${ }^{[28,29]} \mathrm{A}$ voltage of $10 \mathrm{kV}$, a current of $0.05 \mu \mathrm{A}$ and a needle tip-to-ground collector distance of $20 \mathrm{~cm}$ were selected as processing conditions after optimization. An

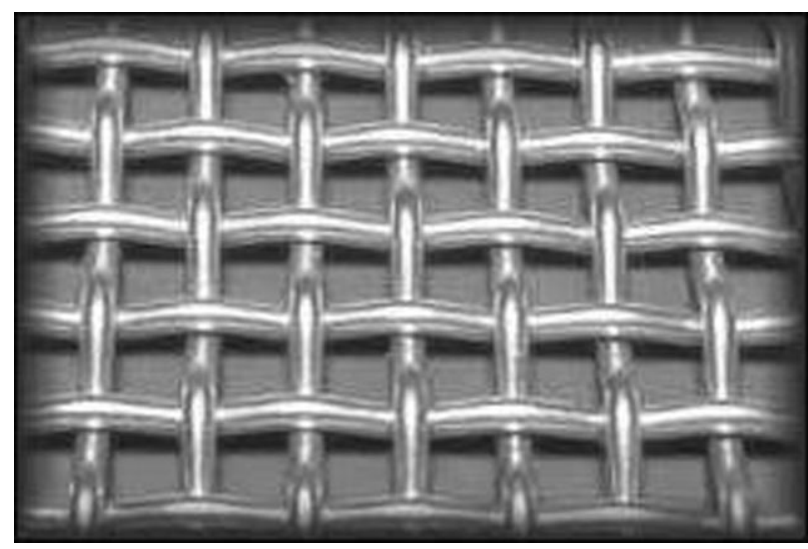

Figure 1. Photograph of the chess-like wire net used to collect the electrospun patterned nanofiber meshes (P-NFM).

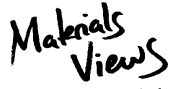

www.MaterialsViews.com
Macromol. Biosci. 2011, 11, 978-987

(C) 2011 WILEY-VCH Verlag GmbH \& Co. KGaA, Weinheim 
electroconductive chess-like wire net with $1.0 \mathrm{~mm}$ spacing between two consecutive wires was used as the collector (Figure 1).

\section{Characterization of Patterned Nanofiber Meshes}

Patterned electrospun PCL nanofiber meshes were gold sputtercoated (model SC502, Fisons Instruments, England) for $2 \mathrm{~min}$ at $15 \mathrm{~mA}$. Samples were analyzed using a scanning electron microscope (model S360, Leica, Cambridge, England). Micrographs were recorded at $15 \mathrm{kV}$ with magnifications ranging from 100 to 5000 times.

The surface topography of the patterned electrospun PCL nanofiber meshes was assessed from the non-contact profilometry using an interferometer profiler (model NT1100, Wyko-Veeco, Tucson, AZ) equipped with WycoVision 32 analytical software. Topographic roughness parameters $R_{\mathrm{a}}$ (roughness average) and $R_{\mathrm{q}}$ [root mean square (RMS) roughness] were determined for each sample. Each sample was evaluated at three randomly selected and representative specimen locations.

\section{Expansion, Seeding and Osteogenic Differentiation of hBMSCs}

hBMSCs (Biopredic International, France) were isolated and characterized according to the method established by Delorme and Charbord. ${ }^{[32]}$ hBMSCs were expanded in basal medium consisting of Dulbecco's modified Eagle's medium (DMEM; Sigma-Aldrich, Germany) supplemented with $10 \%$ heat-inactivated fetal bovine serum (FBS; Biochrom AG, Germany) and 1\% antibiotic/antimyotic solution (final concentration of penicillin 100 units $\cdot \mathrm{mL}^{-1}$ and streptomycin $100 \mu \mathrm{g} \cdot \mathrm{mL}^{-1}$ (Gibco, GB). Cells were cultured in a $5 \% \mathrm{CO}_{2}$ incubator at $37^{\circ} \mathrm{C}$.

Before the in vitro studies, the random and patterned PCL nanofiber meshes were cut into samples with areas of approximately $1 \mathrm{~cm}^{2}$ and sterilized by UV irradiation over $1 \mathrm{~h}$ on each side of the mesh. Confluent hBMSCs at passage 2 were harvested for seeding onto the patterned PCL nanofiber meshes at a density of $1.5 \times 10^{5}$ cells $\cdot \mathrm{cm}^{-2}$ of the nanofiber mesh. Random PCL nanofiber meshes were used as controls in the study. The nanofiber mesh/ hBMSCs constructs were left for $48 \mathrm{~h}$ under agitation (orbital shaker), allowing cells to arbitrarily colonize the entire surface of the mesh. After the seeding was completed, the constructs were cultured for 7 , 14 and $21 \mathrm{~d}$ under static conditions, in standard osteogenic differentiation medium (basal medium supplemented with $50 \mu \mathrm{g} \cdot \mathrm{mL}^{-1}$ ascorbic acid, $10^{-2} \mathrm{M} \beta$-glicerophosphate and $10^{-7} \mathrm{M}$ dexamethasone).

\section{Cell Morphology and Distribution}

After each culture period, the constructs for fluorescence microscopy analysis were fixed in $10 \%$ formalin solution neutral buffer (SigmaAldrich, Germany) for 30 min and maintained in phosphate-buffered saline (PBS) until further use. The nucleus and the cytoskeleton actin filaments of the cells were fluorescently labeled with 4,6-diamidino2-phenyindole dilactate (DAPI; Sigma, USA; dilution 1:1000) and phalloidin-tetramethylrhodamine B isothiocyanate (Sigma, USA; dilution 1:100), respectively, and the constructs analyzed using a fluorescence microscope (Zeiss, Germany).
For scanning electron microscopy (SEM) evaluation, the samples were previously fixed with $2.5 \%$ glutaraldehyde (Sigma, USA) in phosphate buffer saline solution (Sigma, USA) for $1 \mathrm{~h}$ at $4{ }^{\circ} \mathrm{C}$, and then dehydrated through an increasing series of ethanol concentrations and left to dry overnight. Finally, they were gold sputtercoated (sputter coater model SC502, Fisons Instruments, England) and analyzed by SEM (model S360, Leica, Cambridge, England).

\section{Cell Viability and Proliferation (MTS Assay and DNA Content)}

Cell viability for each culturing time was determined using the CellTiter 96 Aqueous One Solution Cell Proliferation Assay (Promega, USA). This assay is based on the bioreduction of a tetrazolium compound, 3-(4,5-dimethylthiazol-2-yl)-5-(3-carboxymethoxyphenyl)-2-(4-sulfofenyl)-2H-tetrazolium (MTS), into a water soluble brown formazan product. This conversion is accomplished by $\mathrm{NADPH}$ or NADH production by the dehydrogenase enzymes in metabolically active cells. The absorbance was measured at $490 \mathrm{~nm}$ using a microplate reader (Synergie HT, Bio-Tek, USA), being related to the quantity of formazan product and directly proportional to the number of living cells in the constructs. Three samples of each nanofiber mesh per time point were characterized.

Cell proliferation was quantified by the total amount of doublestranded DNA along the culturing time. Quantification was performed using the Quant-iT PicoGreen dsDNA Assay Kit (Invitrogen, Molecular Probes, Oregon, USA), according to the instructions of the manufacturer. Briefly, cells in the construct were lyzed by osmotic and thermal shock and the supernatant used for the DNA quantification assay. A fluorescent dye, PicoGreen, was used because of its high sensitivity and specificity to double-stranded DNA. The fluorescence of the dye was measured at an excitation wavelength of $485 / 20 \mathrm{~nm}$ and at an emission wavelength of 528/20 nm, in a microplate reader (Synergie HT, Bio-Tek, USA). Triplicates were carried out for each sample and per culturing time. The DNA concentration for each sample was calculated using a standard curve (DNA concentration ranging from 0.0 to $1.5 \mu \mathrm{g} \cdot \mathrm{mL}^{-1}$ ) relating the quantity of DNA and fluorescence intensity.

\section{Alkaline Phosphatase Quantification and Immunodetection of Bone-Specific Proteins}

The concentration of alkaline phosphatase (ALP) was determined for all time culture periods, using the same samples used for DNA quantification. Briefly, the activity of ALP was assessed using the pnitrophenol assay. Nitrophenyl phosphate disodium salt (pnPP; Fluka BioChemika, Austria), which is colorless, is hydrolyzed by alkaline phosphatase at $\mathrm{pH}=10.5$ and a temperature of $37^{\circ} \mathrm{C}$ to form free $p$-nitrophenol, which is yellow. The reaction was stopped by the addition of $2 \mathrm{M} \mathrm{NaOH}$ (Panreac Quimica, Spain) and the absorbance read at $405 \mathrm{~nm}$ in a microplate reader (Bio-Tek, Synergie $\mathrm{HT}$, USA). Standards were prepared with $10 \mu \mathrm{mol} \cdot \mathrm{mL}^{-1}$ p-nitrophenol (pNP; Sigma, USA) solution, to obtain a standard curve ranging from 0 to $0.3 \mu \mathrm{mol} \cdot \mathrm{mL}^{-1}$. Triplicates of each sample and standard were made, and the ALP concentrations read off from the standard curve.

The osteogenic phenotype of hBMSCs seeded onto the patterned PCL nanofiber meshes was also assessed by the immunodetection 
of bone-specific proteins. Immunocytochemistry was performed following the streptavidin/biotin peroxidase complex approach (R.T.U. Vectastain Universal Elite ABC kit; Vector Laboratories Inc., Burlingame, CA), using a rabbit polyclonal antibody against osteopontin (Abcam Ltd., Cambridge, UK; dilution 1:1500), a mouse monoclonal antibody against osteocalcin (clone OC4-30, Abcam Ltd., Cambridge, UK; dilution 1:100) and a rabbit polyclonal antibody against bone sialoprotein II (Chemicon International Inc., Germany; dilution 1:2500). Prior to the immunocytochemistry procedure, constructs were fixed in $10 \%$ formalin solution neutral buffer (Sigma-Aldrich, Germany) for $30 \mathrm{~min}$ and maintained in phosphate buffer saline (PBS) until further use. The constructs were treated with $0.3 \%$ hydrogen peroxide in methanol for $30 \mathrm{~min}$ to inactivate the endogenous peroxidases. After washing with PBS, the constructs were blocked with $2.5 \%$ normal horse serum for $20 \mathrm{~min}$ at room temperature to avoid unspecific reactions. Primary antibodies were incubated overnight at $4{ }^{\circ} \mathrm{C}$. Negative controls were set in the absence of primary antibody incubation. After washing in PBS, the samples were incubated for $30 \mathrm{~min}$ with biotinylated secondary antibody anti-rabbit/mouse IgG, followed by incubation with streptavidin-peroxidase complex (Elite $A B C$ Reagent). The immune reaction was visualized using $D A B$ as a chromogen (DAB Substrate Reagent from Peroxidase Substract Kit; Vector Laboratories Inc, Burlingame, CA).

\section{RNA Isolation and Real-Time Quantitative Polymerase Chain Reaction (qPCR)}

Total RNA from the constructs was extracted using the Trizol (Invitrogen, Life Technologies Inc., UK) method, according to the manufacturer's directions. Briefly, at each culturing time, the constructs were washed with PBS, immersed in Trizol and stored at $-80^{\circ} \mathrm{C}$ until further use. Proteins were removed with chloroform extraction, and the RNA pellets were washed once with isopropyl alcohol and once with $70 \%$ ethanol. The total RNA pellets were reconstituted in RNAse-free water (Gibco, Invitrogen, UK).

Reverse transcriptase (RT)-PCR was performed according to the protocol from the iScript cDNA synthesis kit (BioRad, Hercules, CA, USA). Briefly, a reaction mixture consisting of $1 \mathrm{X}$ iScript Reaction Mix, $1 \mu \mathrm{L}$ iScript Reverse Transcriptase, RNA template (up to $1 \mu \mathrm{g}$ total RNA) and nuclease-free water was prepared, in $20 \mu \mathrm{L}$ of total volume. The single-strand cDNA synthesis occurred by incubating the complete reaction mixture for $5 \mathrm{~min}$ at $25^{\circ} \mathrm{C}$, followed by $30 \mathrm{~min}$ at $42^{\circ} \mathrm{C}$, and was then terminated by an incubation at $85^{\circ} \mathrm{C}$ for $5 \mathrm{~min}$.

Amplification of the target CDNA for real-time PCR quantification was performed according to the manufacturer, using $2 \mu \mathrm{L}$ of RT cDNA products, $10^{-6} \mathrm{M}$ of each primer (bone-specific primer sets listed in Table 1), 1X iO SYBR Green Supermix (BioRad, Hercules, CA, USA) and nuclease-free water, in a final volume of $25 \mu \mathrm{L}$. 44 cycles of denaturation $\left(95^{\circ} \mathrm{C}, 10 \mathrm{~s}\right)$, annealing (temperature dependent on the gene, $30 \mathrm{~s})$ and extension $\left(72{ }^{\circ} \mathrm{C}, 30 \mathrm{~s}\right)$ were carried out in a gradient thermocycler MiniOpticon real-time PCR detection system (BioRad, Hercules, CA, USA) for all genes. The transcripts' expression data were normalized to the housekeeping gene glyceraldehyde-3-phosphate-dehygrogenase (GAPDH) and the relative quantification calculated by the $\triangle C_{\mathrm{T}}$ method.

\section{Statistical Analysis}

Statistical analysis was performed using the SPSS statistic software (Release 15.0.0 for Windows). Firstly, a Shapiro-Wilk test was used to ascertain the data normality. The results indicated that nonparametric tests should be used for all comparisons. A MannWhitney U test was performed to analyze the effect of the random and patterned electrospun nanofibrous structures on the hBMSCs-

Table 1. Primers list of osteogenic markers.

$\begin{array}{lc}\text { Gene } & \\ \text { ALP } & \text { sense } \\ \text { OPtisense } & \text { sense } \\ \text { OP } & \text { antisense } \\ \text { BSP } & \text { sense } \\ \text { OC } & \text { antisense } \\ \text { Runx2 } & \text { sense } \\ \text { Osterix } & \text { antisense } \\ & \text { sense } \\ \text { GAPDH } & \text { antisense } \\ & \text { sense } \\ & \text { antisense } \\ & \text { sense } \\ \end{array}$

Primer sequences

$\left(5^{\prime}-3^{\prime}\right)$
$T_{\mathrm{m}}$ $\left[{ }^{\circ} \mathrm{C}\right]$ 60.0

GGCAGCGAGGTAGTGAAGAG

TTCCAGACCAGCAGCACTC

CAGCGTCAACACCATCATTC

CCСTTTACAAGCACTAATGG

ACACTGGGCAGACAGTCAG

ACAGTCAGCCGCATCTTCTT

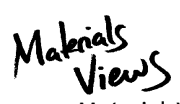


derived phenotype (cellular proliferation and ALP quantification) and osteogenic genotype. $P$ values lower than 0.01 were considered statistically significant.

\section{Results and Discussion}

\section{Morphological Characterization of Patterned Nanofiber Meshes}

Typically, a random distribution of nanofibers is obtained in a static flat collector, caused by the chaotic motion of polymeric solution during the electrospinning process [Figure 2(A)]. However, when a metallic conducting wire net was used as collector, two distinct areas of nanofiber deposition [Figure 2(B)], reproducing the architecture of the collector used, were obtained. ${ }^{[29,30]}$ The fibers appeared aligned and collapsed on the electroconductive wires of the collector and, consequently, where the electric field was more intense [Figure 2(C)]. This area was characterized by significantly larger fiber densities, due to preferential deposition and fiber agglomeration, which is believed to be caused by the excess of residual solvent during the deposition process. Consequently, in this area of parallel/ uniaxial alignment, the average roughness, determined by interferometric optical profilometry, was significantly smaller $\left(R_{\mathrm{a}}=789.68 \mathrm{~nm}\right)$ [Figure $2(\mathrm{G})$ ], when compared to the random nanofiber meshes (PCL NFM) $\left(R_{\mathrm{a}}=1.93 \mu \mathrm{m}\right)$ [Figure 2(E)]. In the spacing between the wires, the nanofiber deposition followed an orthogonally aligned pattern and had a lower density [Figure 2(D)]. The roughness parameter analysis revealed a smooth surface in the orthogonal alignment area $\left(R_{\mathrm{a}}=1.79 \mu \mathrm{m}\right)$ [Figure 2(H)], when compared with the typical random nanofiber meshes [Figure 2(E)].

It is well known that materials with ordered microstructures and patterns may possess specific interest functions useful in numerous applications, such as micro- electronic, photonic and biomedical applications. ${ }^{[33]}$ As previously mentioned and herein described, our group has demonstrated the possibility to electrospin nanofiber meshes with controlled microstructure and pattern structures, demonstrating their potential for biomedical applications. ${ }^{[28,29]}$ In fact, these patterned structures were initially developed in an attempt to control the morphological and, consequently, the mechanical properties of the nanofiber meshes. It was demonstrated that P-NFM presents a tensile modulus of $2.0 \pm 0.2 \mathrm{MPa}$, which was lower than expected due to the high degree of fiber alignment. ${ }^{[29]}$ However, the orthogonal fiber alignment in the mesh associated with uniaxial fiber alignment, transversal to the testing of the main tensile test direction, explained the observed value of the modulus. The transversely aligned fibers could control the strain in the tensile test, causing more compliant behavior of the P-NFM. Even so, the semi-crystalline property of PCL is likely to have a beneficial effect on the structural integrity of the scaffold, maintaining the size and the shape of the original P-NFM, without observable macroscopic shrinkage.

\section{Phenotypic Characterization of Differentiated hBMSCs on Patterned Nanofiber Meshes}

Various studies have demonstrated that electrospun PCL scaffolds exhibit optimal structural integrity and support desirable cellular proliferation and differentiation (MSCs differentiated into osteoblastic- and chondrocytic-like cells) in vitro. ${ }^{[9,20,21,34,35]}$ In addition, 3D porous networks, composed of PCL and collagen (COL) (1:1), produced by the multi-layered organization of electrospun nanofiber membranes appeared to support hBMSCs attachment. ${ }^{[36]}$ Subcutaneous implantation of the cultured construct into nude mice demonstrated good integration with the surrounding tissues and neovascularisation. Electrospun PCL scaffolds seeded with autologous mesenchymal stem
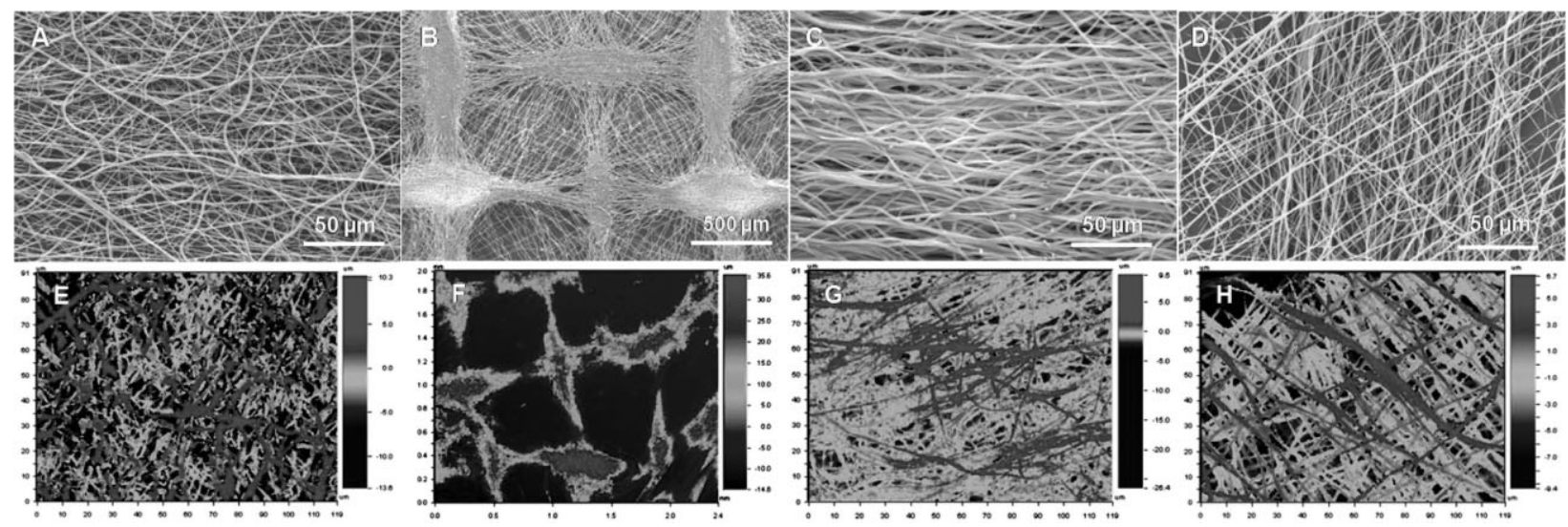

Figure 2. SEM micrographs (A-D) and optical profilometric images $(E-H)$ of the typical random $(A$ and $E)$ and patterned $(B$ and $F)$ nanofiber meshes. P-NFM comprise areas of parallel/uniaxial ( $C$ and $G$ ) and orthogonal ( $D$ and $H$ ) alignment of the fibers. 
cells (MSCs) were studied as bone grafts for the regeneration of the omenta of rats. ${ }^{[37]}$ The cell/scaffold constructs were cultured under osteogenic supplementation, in a rotating bioreactor, before implantation. Those studies show the interest of electrospun nanofibrous scaffolds as bone grafts.

The search for surfaces and structures having a strong, well-defined, beneficial influence on cell growth is an active subject in contemporary research. ${ }^{[3,38]}$ In fact, it has been demonstrated that cells are sensitive to the topography of the supporting surface, although the exact reasons for this observation are unclear. In our previous studies with patterned nanofiber meshes, direct contact tests with human osteoblast-like cells demonstrated preferential cell adhesion to regions of random/orthogonal fiber alignment, although some cells were aligned with the regions of parallel orientation. ${ }^{[28,29]}$ Initially, in the present study, hBMSCs were seeded and cultured over $7 \mathrm{~d}$ on random and patterned PCL nanofiber meshes (P-NFM) to verify the influence of the nanofiber mesh topography on cell morphology and distribution. SEM micrographs demonstrated that undifferentiated cells responded differently to the different areas of P-NFM (Figure 3). In orthogonally distributed nanofibers, cells presented the typical fibroblastic morphology of undifferentiated hBMSCs [Figure 3(A)]. In the area of parallel/ uniaxial alignment, hBMSCs attached and spread along the aligned nanofibers of the patterned fiber meshes [Figure 3(B)]. Thus, the patterned nanofibrous scaffolds created in this study were found to dictate cellular morphology, with cell polarity following the established fiber direction. These observations are supported by a recent study showing that hMSCs maintained their phenotypic shape when seeded on randomly oriented scaffolds, and a guided growth with actin organization dictated by the prevailing nanofiber orientation of aligned nanofiber meshes. ${ }^{[39]}$ Overall, the results in the literature demonstrate that highly oriented electrospun PCL nanofibers are capable of supporting cell attachment and the proliferation of hMSCs.

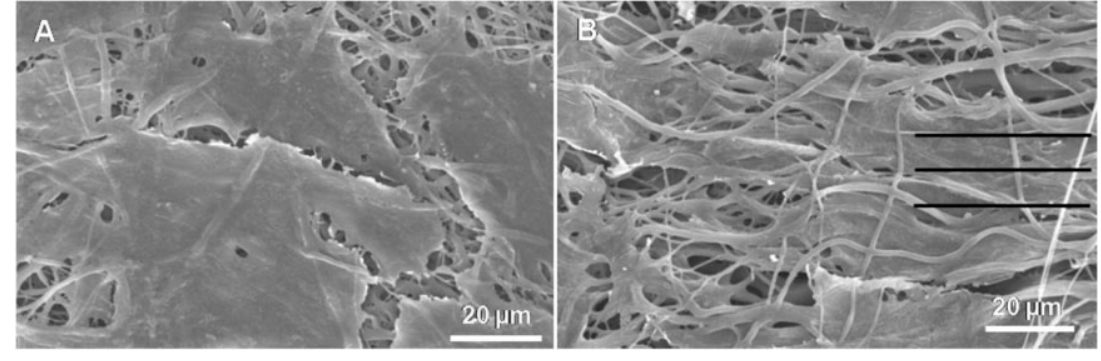

Figure 3. SEM micrographs of undifferentiated hBMSCs cultured on patterned nanofiber meshes over 7 days, at the random orthogonal area of alignment $(A)$ and at the parallel uniaxial fibers (orientation is indicated by black lines) (B).
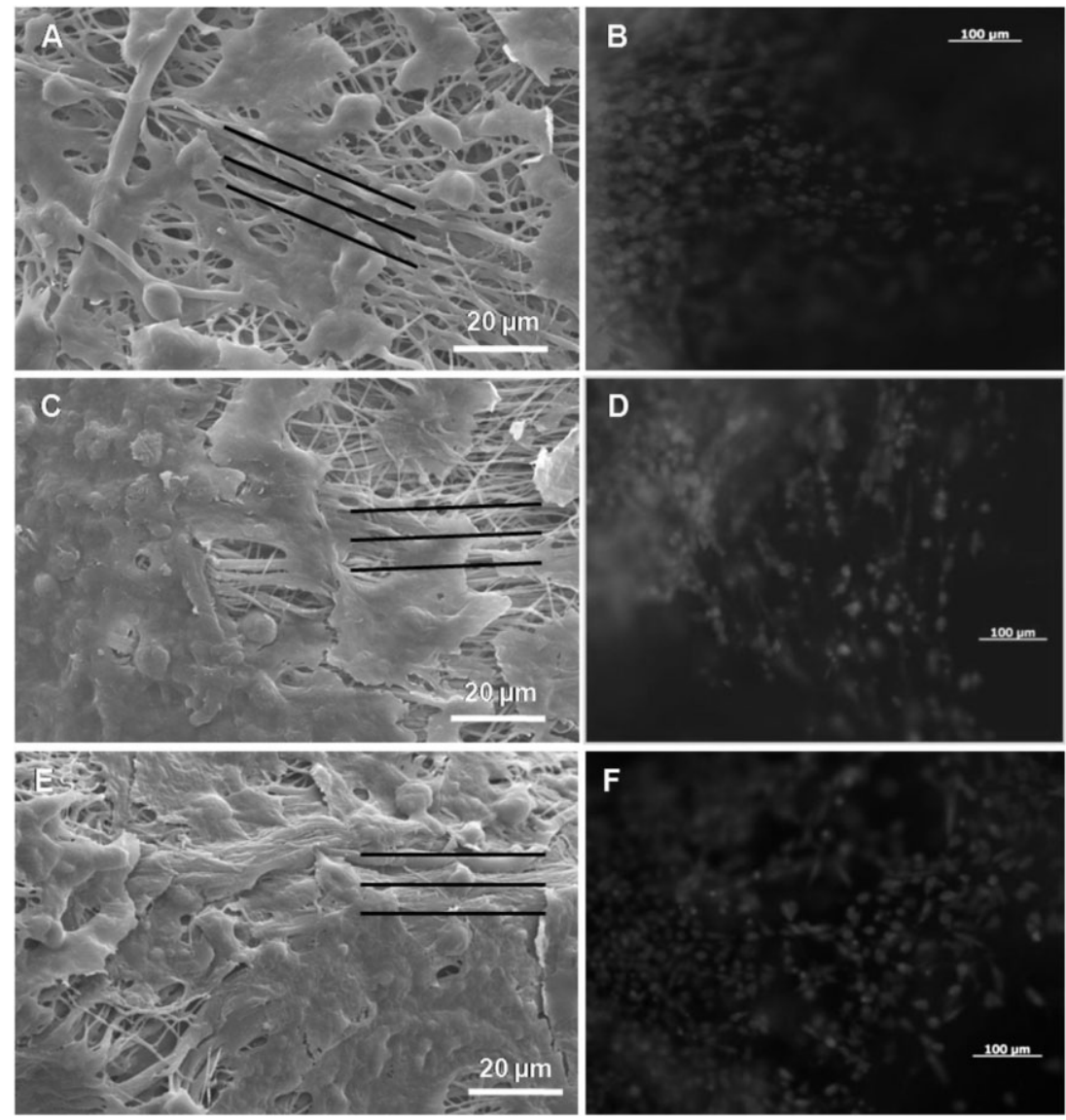

Figure 4. SEM micrographs ( $A, C$ and $E$ ) and fluorescence images ( $B, D$ and $F)$ of hBMSCs induced to differentiate into the osteogenic lineage after 7 ( $A$ and $B), 14$ ( $C$ and $D)$ and 21 $(E$ and $F$ ) days of culture. Black lines represent the orientation of parallel/uniaxial aligned fibers.

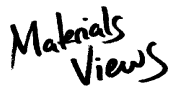

www.MaterialsViews.com
Macromol. Biosci. 2011, 11, 978-987

(C) 2011 WILEY-VCH Verlag GmbH \& Co. KGaA, Weinheim
Considering the previous findings, hBMSCs were induced to differentiate into the osteogenic lineage, with the aim of understanding the influence of the P-NFM on the osteogenic differentiation of hBMSCs, when compared with the typical PCL NFM. SEM and fluorescence microscopy characteriza- 
tion of cell adhesion and morphology showed a cell response to the topography of the P-NFM, being spread in the area of nanofiber parallel/uniaxial alignment [Figure 4(A)-4(D)]. The induction of cell alignment by the nanofibers was only observed in the outer regions of the PNFM, where a lower density of cells was present, due to the increment of cell mass in the center of the mesh. For longer culture periods, hBMSCs started to differentiate into the osteoblastic lineage and tended to show a more polygonal shape [Figure 4(E)]. This increment in the cell numbers was confirmed by the cellular proliferation assay, by quantification of the double strand DNA content (Figure 5). The DNA content of hBMSCs, induced to differentiate into the osteogenic lineage, increased progressively with culture time on P-NFM. In fact, it was only found a highly significant difference $(p<0.00001)$ between the PCL NFM and P-NFM for $7 \mathrm{~d}$ of culture in terms of DNA concentration.

Osteoblastic differentiation of hBMSCs was pursued on P-NFM by the quantification of the alkaline phosphatase (ALP). Indeed, ALP production is a distinctive biochemical indicator of the presence of osteoblasts, since MSCs produce negligible amounts of this enzyme. It can be observed in Figure 6 that the ALP concentration values increase progressively with time in the culture of hBMSCs, when seeded on the patterned nanofiber meshes, reaching a median maximum value at $14 \mathrm{~d}$ of culture under osteogenic differentiation conditions. The corresponding values of the ALP concentration, for PCL NFM, maintained a constant value with time. Additionally, it was only found a highly significant difference $(p<0.00001)$ between the PCL NFM and P-NFM for $7 \mathrm{~d}$ of culture, in terms of ALP concentration.

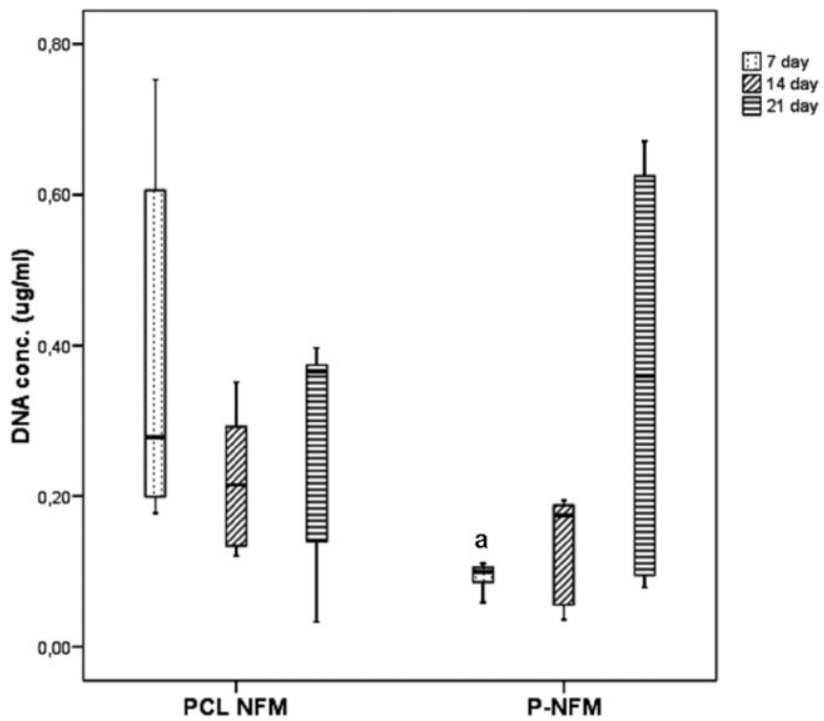

Figure 5. Box plot of DNA content of hBMSCs induced to differentiate into the osteogenic lineage over 21 days, cultured on random and patterned nanofiber meshes. Data were analyzed by the non-parametric method of the Mann-Whitney $U$ test. $a$, $p<0.01$.

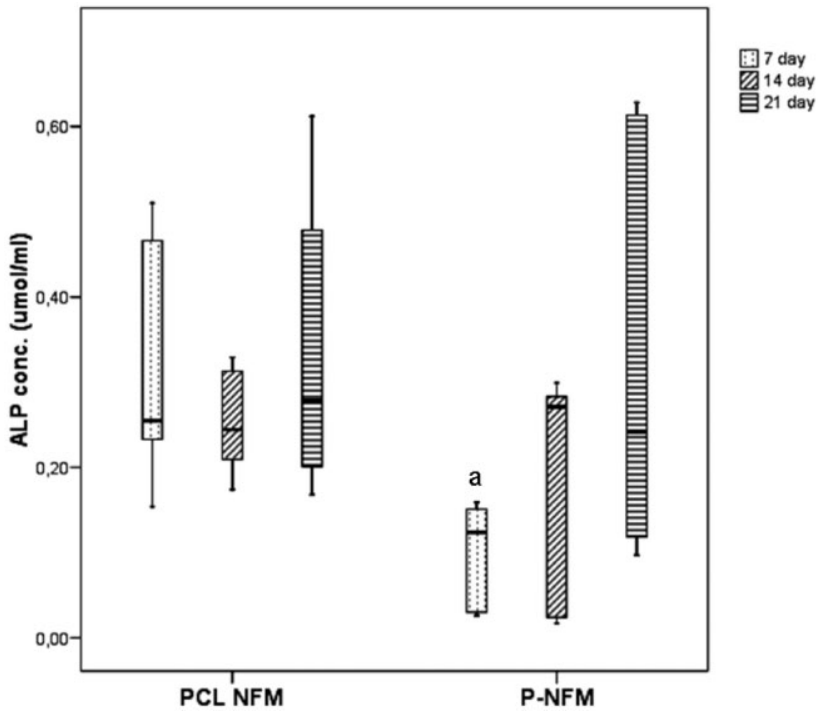

Figure 6. Box plot of ALP quantification from hBMSCs induced to differentiate into the osteogenic lineage over 21 days, cultured on random and patterned nanofiber meshes. Data were analyzed by the non-parametric method of the Mann-Whitney $U$ test. $a$, $p<0.01$.

Immuncytochemistry against some bone-specific proteins, namely osteopontin, osteocalcin and bone sialoprotein, was performed to confirm the osteogenic phenotype of hBMSCs seeded onto the P-NFM. Photomicrographs showed a progressive expression of specific osteoblastic glycoproteins (i.e., osteopontin and bone sialoprotein) on hBMSCs/ patterned nanofiber mesh constructs along the culturing period (Figure 7), indicating their capability to deposit mineralized extracellular matrix (ECM) mainly in the areas of parallel/uniaxial alignment of the nanofibers. In fact, these phosphorylated glycoproteins are also present in the ECM of bone. The deposition of mineralized ECM was also confirmed by the immunodetection of osteocalcin protein, which binds strongly to apatite and calcium. Besides that, the concomitant increment of the enzyme alkaline phosphatase (ALP) until $14 \mathrm{~d}$ of hBMSCs culture also confirmed the osteoblastic differentiation and mineralization of hBMSCs on patterned nanofiber meshes, because this enzyme catalyzes the splicing of phosphate from non-phosphoric esters, constituting an early biochemical marker of osteogenesis and deposition of mineralized ECM. These observations are corroborated by a study where hybrid random nanofibrous scaffolds, consisting of PCL, poly(vinyl alcohol) (PVA) and chitosan, were used to culture MSCs and successfully sustained the induced differentiation into osteoblasts. ${ }^{[40]}$

\section{Genotypic Characterization of Differentiated hBMSCs on Patterned Nanofiber Meshes}

Complementary to previous biological data, the differentiation level of seeded hBMSCs on P-NFM was assessed by 


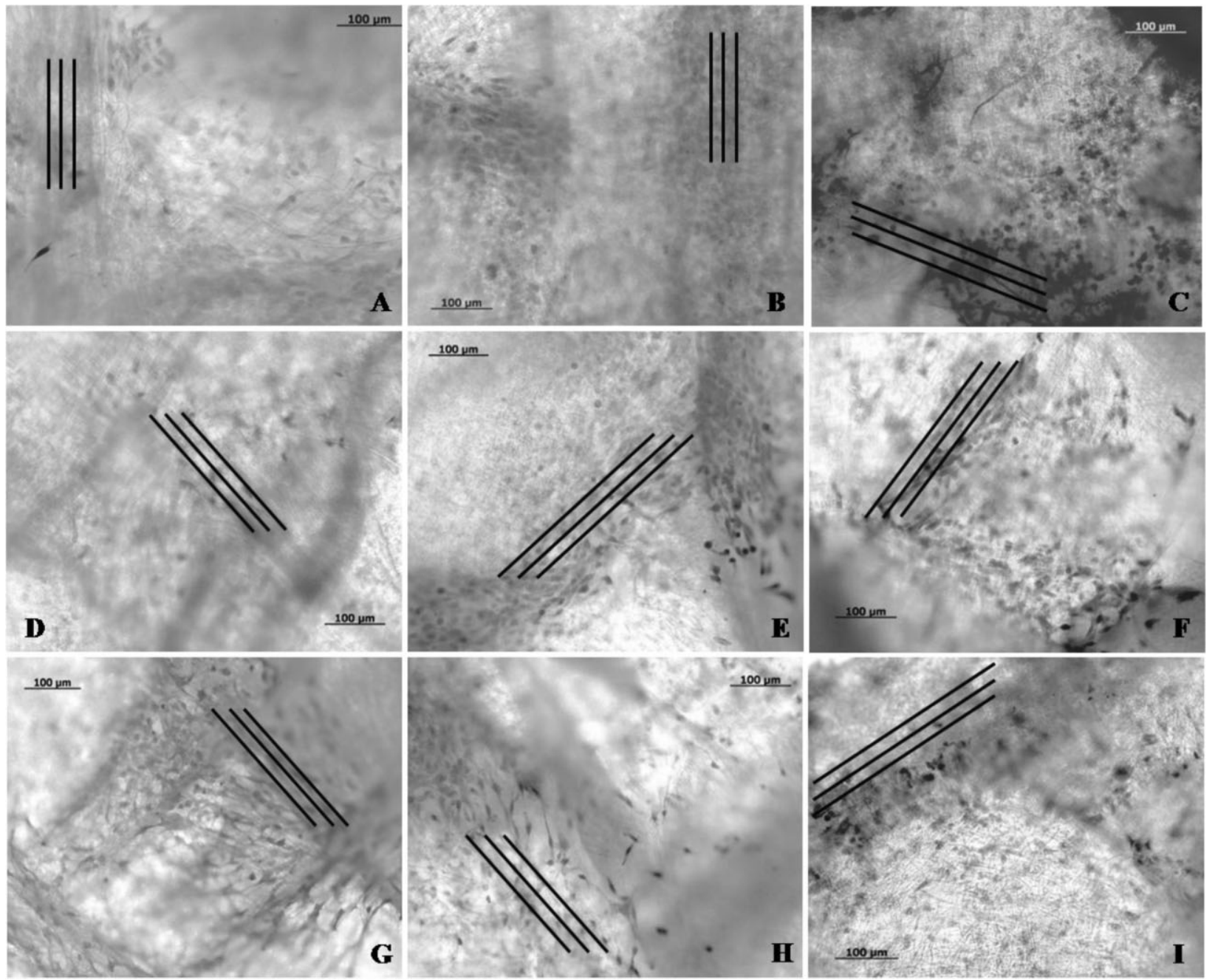

Figure 7. Optical images of immunodetected osteogenic markers, namely osteopontin (A-C), bone sialoprotein (D-F) and osteocalcin (G-I), expressed by hBMSCs induced to differentiate into the osteogenic lineage and cultured on patterned nanofiber meshes over 7 (A, D and G), 14 (B, E and H) and 21 (C, F and I) days. Black lines represent the orientation of parallel/uniaxial aligned fibers.

quantitative PCR of mRNA transcripts of some bone-specific genes. The relative expression of those genes was normalized against the housekeeping gene GAPDH and compared to hBMSCs cultured and differentiated on PCL NFM. From the analysis of Figure 8, it was observed that some transcript levels, namely Alkaline Phosphatase, Runx2 and Osterix, showed a tendency to be increasingly expressed on P-NFM, during $21 \mathrm{~d}$ of culture. The remaining transcripts, specifically the Bone Sialoprotein and the Osteocalcin, were expressed at stable levels during the period in culture, with the exception of Osteopontin, which presented the highest expression at $14 \mathrm{~d}$. Recently, the expression pattern of osteoblastic markers during the differentiation of embryonic stem (ES) cells was reported. ${ }^{[41]}$ Three periods were defined, namely a proliferative phase, followed by a period of matrix deposition and the mineralization phase. At the end of the matrix deposition phase and the beginning of the mineralization phase, Osteopontin mRNA was expressed. Bone Sialoprotein was expressed during the mineralization phase, corresponding to the presence of mature osteoblasts. Finally, Osteocalcin mRNA was expressed at a very high level and so was designed as the essential marker of the mineralization phase. Thus, considering our results and the differences in the cell type used, the most important genes involved in the mineralization process (i.e., Osteopontin, Bone Sialoprotein and Osteocalcin genes) were constitutively expressed, confirming the matrix deposition and mineralization by hBMSCs cultured and differentiated on P-NFM. The main specific transcription factors involved in the osteogenesis were also quantified by $\mathrm{qPCR}$, namely the core binding factor $\alpha 1 /$ runt-related gene (Cbfa1/Runx2) and Osterix (Osx). Cbfa1/Runx2 has been shown to preferentially initiate two steps of the differentiation process, stem cells

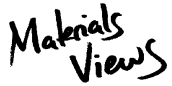

www.MaterialsViews.com
Macromol. Biosci. 2011, 11, 978-987

(C) 2011 WILEY-VCH Verlag GmbH \& Co. KGaA, Weinheim

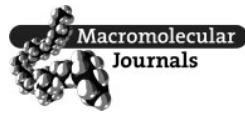




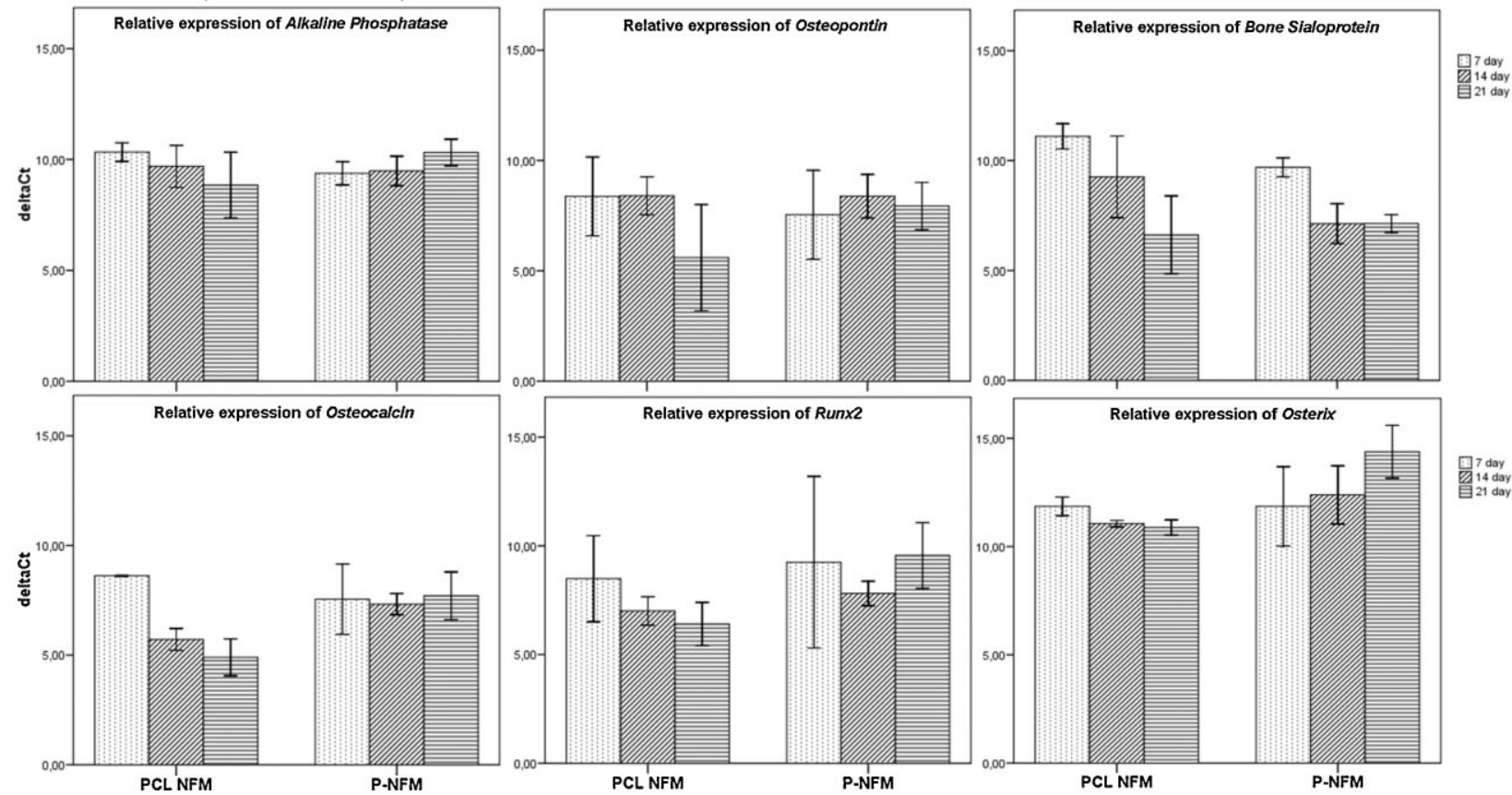

Figure 8. Relative expression of bone-specific transcripts, namely Alkaline Phosphatase, Osteopontin, Bone Sialoprotein, Osteocalcin, Runx2 and Osterix, by hBMSCs induced to differentiate into the osteogenic lineage over 21 days, cultured on random and patterned nanofiber meshes. The expression of these genes was normalized against the housekeeping gene GAPDH and calculated by the $\triangle C_{\mathrm{T}}$ method.

into preosteoblasts and preosteoblasts into osteoblasts, whereas Osterix acts only during the last preosteoblast/ osteoblast stage. ${ }^{[42,43]}$ Our results showed that Osterix was the bone-specific transcript with the highest expression relative to the housekeeping gene (GAPDH), accompanied by the progressive expression of Runx2, corroborating the successful osteogenic differentiation of hBMSCs on P-NFM. Altogether, the results from phenotypic and genotypic characterization demonstrated the effectiveness of P-NFM on the differentiation of hBMSCs into osteoblastic cells and, consequently, in the deposition of mineralized ECM. Although no significant differences were observed between the two types of electrospun meshes, the complex ordered microtopography could promote a favorable biological response, such as cell guidance and proliferation. Recently, a study demonstrated that ligament fibroblasts cultured on aligned nanofibrous meshes produce more collagen than random meshes. ${ }^{[4]}$ Indeed, material structures with parallel orientation or patterns of different ordering may have specific biological performance in tissue engineering. ${ }^{[38,44,45]}$ Therefore, it is hypothesized that by creating an initial synthetic ECM architecture, cell-formed ECM will be deposited along the pre-defined fiber direction in greater amounts in these patterned nanofibrous scaffolds, facilitating the formation of a neo-tissue with enhanced functional characteristics. The application of those nanofibrous pattern scaffolds for the regeneration of complex hierarchical and highly organized functional tissues, involving more than one cell community, may provide helpful cues enabling its self-assembly.

\section{Conclusion}

The phenotype of hBMSCs was sensitive to the unique microtopography of P-NFM, inducing cell orientation along the uniaxially aligned fibers, mainly at earlier culturing periods under basal and osteogenic differentiation conditions. The unique topography of the patterned scaffold sustained the deposition of mineralized extracellular matrix, as observed by the immunodetection of osteoblast-specific glycoproteins (i.e., osteopontin and bone sialoprotein), as well as of osteocalcin protein, associated with an increased ALP concentration. Additionally, the progressive expression of bone-specific transcripts confirmed the osteogenic genotype of cultured hBMSCs on PNFM. Our observations indicate that the presence of ordered microstructures and patterns supports the development of bony-like engineered substitutes.

Acknowledgements: This work was partially supported by the European Integrated Project GENOSTEM (LSH-STREP-CT-2003503161) and the European Network of Excellence EXPERTISSUES (NMP3-CT-2004-500283). The authors also acknowledge the Portuguese Foundation for Science and Technology for the project 
Naturally Nano (POCI/EME/58982/2004) and the PhD grant of A. Martins (SFRH/BD/24382/2005).

Received: January 12, 2011; Revised: March 2, 2011; Published online: April 11, 2011; DOI: 10.1002/mabi.201100012

Keywords: biodegradable; electrospinning; mesenchymal stem cells; patterned nanofiber meshes; tissue engineering

[1] O. P. Pham, U. Sharma, A. G. Mikos, Tissue Eng. 2006, 12, 1197.

[2] N. Ashammakhi, A. Ndreu, A. M. Piras, L. Nikkola, T. Sindelar, H. Ylikauppila, A. Harlin, M. E. Gomes, N. M. Neves, E. Chiellini, F. Chiellini, V. Hasirci, H. Redl, R. L. Reis, J. Nanosci. Nanotechnol. 2007, 7, 862 .

[3] J. Lannutti, D. Reneker, T. Ma, D. Tomasko, D. Farson, Mater. Sci. Eng. C: Biomimet. Supramol. Syst. 2007, 27, 504.

[4] A. Martins, J. V. Araujo, R. L. Reis, N. M. Neves, Nanomedicine 2007, 2, 929.

[5] A. Martins, R. L. Reis, N. M. Neves, Int. Mater. Rev. 2008, 53 , 257.

[6] B. M. Min, G. Lee, S. H. Kim, Y. S. Nam, T. S. Lee, W. H. Park, Biomaterials 2004, 25, 1289.

[7] C. Y. Xu, R. Inai, M. Kotaki, S. Ramakrishna, Tissue Eng. 2004, 10,1160

[8] W. J. Li, K. G. Danielson, P. G. Alexander, R. S. Tuan, J. Biomed. Mater. Res. A 2003, 67A, 1105.

[9] M. A. da Silva, A. Crawford, J. Mundy, A. Martins, J. V. Araújo, P. V. Hatton, R. L. Reis, N. M. Neves, Tissue Eng. Part A 2009, 15, 377.

[10] K. Fujihara, M. Kotaki, S. Ramakrishna, Biomaterials 2005, 26, 4139.

[11] J. V. Araujo, A. Martins, I. B. Leonor, E. D. Pinho, R. L. Reis, N. M. Neves, J. Biomater. Sci., Polym. Ed. 2008, 19, 1261.

[12] M. Shin, O. Ishii, T. Sueda, J. P. Vacanti, Biomaterials 2004, 25 , 3717.

[13] W. J. Li, R. Tuli, X. X. Huang, P. Laquerriere, R. S. Tuan, Biomaterials 2005, 26, 5158.

[14] F. Yang, C. Y. Xu, M. Kotaki, S. Wang, S. Ramakrishna, J. Biomater. Sci., Polym. Ed. 2004, 15, 1483.

[15] M. E. Gomes, R. L. Reis, Int. Mater. Rev. 2004, 49, 261.

[16] D. W. Hutmacher, Biomaterials 2000, 21, 2529.

[17] A. J. Salgado, O. P. Coutinho, R. L. Reis, Macromol. Biosci. 2004, 4, 743.

[18] S. Bajada, I. Mazakova, J. B. Richardson, N. Ashammakhi, J. Tissue Eng. Regen. Med. 2008, 2, 169.
[19] M. F. Pittenger, A. M. Mackay, S. C. Beck, R. K. Jaiswal, R. Douglas, J. D. Mosca, M. A. Moorman, D. W. Simonetti, S. Craig, D. R. Marshak, Science 1999, 284, 143.

[20] W. J. Li, R. Tuli, C. Okafor, A. Derfoul, K. G. Danielson, D. J. Hall, R. S. Tuan, Biomaterials 2005, 26, 599.

[21] H. Yoshimoto, Y. M. Shin, H. Terai, J. P. Vacanti, Biomaterials 2003, 24, 2077.

[22] X. J. Xin, M. Hussain, J. J. Mao, Biomaterials 2007, 28, 316.

[23] J. Doshi, D. H. Reneker, J. Electrostat. 1995, 35, 151.

[24] D. Li, Y. N. Xia, Adv. Mater. 2004, 16, 1151.

[25] G. C. Rutledge, S. V. Fridrikh, Adv. Drug Deliv. Rev. 2007, 59 1384.

[26] R. Murugan, S. Ramakrishna, Tissue Eng. 2007, 13, 1845.

[27] A. Martins, S. Chung, A. J. Pedro, R. A. Sousa, A. P. Marques, R. L. Reis, N. M. Neves, J. Tissue Eng. Regen. Med. 2009, 3, 37.

[28] A. Martins, J. Cunha, F. Macedo, R. L. Reis, N. M. Neves, Nanotech 2006, Vol. 2, Boston, USA 2006.

[29] N. M. Neves, R. Campos, A. Pedro, J. Cunha, F. Macedo, R. L. Reis, Int. J. Nanomed. 2007, 2, 433.

[30] D. Zhang, J. Chang, Adv. Mater. 2007, 19, 3662.

[31] D. Zhang, J. Chang, Nano Lett. 2008, 8, 3283.

[32] B. Delorme, P. Charbord, Methods Mol. Med. 2007, 140, 67.

[33] G. M. Whitesides, Nat. Biotechnol. 2003, 21, 1161.

[34] W. J. Li, J. A. Cooper, Jr., R. L. Mauck, R. S. Tuan, Acta Biomater. 2006, 2, 377.

[35] A. Martins, E. D. Pinho, S. Faria, I. Pashkuleva, A. P. Marques, R. L. Reis, N. M. Neves, Small 2009, 5, 1195.

[36] S. Srouji, T. Kizhner, E. Suss-Tobi, E. Livne, E. Zussman, J. Mater Sci., Mater. Med. 2008, 19, 1249.

[37] M. Shin, H. Yoshimoto, J. P. Vacanti, Tissue Eng. 2004, 10, 33.

[38] M. J. Dalby, N. Gadegaard, R. Tare, A. Andar, M. O. Riehle, P. Herzyk, C. D. Wilkinson, R. O. Oreffo, Nature Mater. 2007, 6, 997.

[39] W. J. Li, R. L. Mauck, J. A. Cooper, X. Yuan, R. S. Tuan, J. Biomech 2007, 40, 1686.

[40] Y. Mohammadi, M. Soleimani, M. Fallahi-Sichani, A. Gazme, V. Haddadi-Asl, E. Arefian, J. Kiani, R. Moradi, A. Atashi, N. Ahmadbeigi, Int. J. Art. Organs 2007, 30, 204.

[41] N. I. zur Nieden, G. Kempka, H. J. Ahr, Differentiation 2003, 71 , 18.

[42] H. M. Ryoo, M. H. Lee, Y. J. Kim, Gene 2006, 366, 51.

[43] N. K. Satija, G. U. Gurudutta, S. Sharma, F. Afrin, P. Gupta, Y. K. Verma, V. K. Singh, R. P. Tripathi, Stem Cells Dev. 2007, 16, 7.

[44] C. H. Lee, H. J. Shin, I. H. Cho, Y. M. Kang, I. A. Kim, K. D. Park, J. W. Shin, Biomaterials 2005, 26, 1261.

[45] X. Zong, H. Bien, C. Y. Chung, L. Yin, D. Fang, B. S. Hsiao, B. Chu, E. Entcheva, Biomaterials 2005, 26, 5330. 